\title{
Synthesis of 5-(1,2,3-Triazol-4-yl)-1,3,4-Oxa(thia) diazol-2-Amines as Antimicrobial Agents
}

\author{
B.F. Abdel-Wahab ${ }^{\#}$, Hanan A. Mohamed and Ghada E.A. \\ Awad* \\ Applied Organic Chemistry Department and *Chemistry of \\ Natural and Microbial Products Dept., National Research \\ Center, Dokki, 12622 Giza, Egypt.
}

\begin{abstract}
N EFFICIENT method for the synthesis of new 1,2,3-triazol-4-ylA 1,3,4-oxadiazol-2-amines (4a-c) from phenyliso-thiocyanate and 1,2,3-triazolyl-4-hydrazides (3a-c) through cyclo-desulfurization in the presence of sodium acetate is reported. Also, cyclization of 3a-c with concentrated $\mathrm{H}_{2} \mathrm{SO}_{4}$ afforded the corresponding triazol-4-ylthiadiazoles $(6 a-c)$. The new products were screened for their antimicrobial activities, and compounds; 4a-c showed excellent antimicrobial activities compared with the reference drugs. Also, the minimum inhibitory concentration (MIC) against the tested organisms was determined in which, compounds $2 \mathrm{c}$ and $4 \mathrm{a}$ showed the lowest MIC.
\end{abstract}

Keywords: Triazolyloxadiazoles, Triazolylthiadiazoles, Antimicrobial activity and MIC

1,3,4-Oxadiazoles containing different functional groups have attracted a great deal of attention from synthetic and medicinal chemists that has led to production of novel compounds with improved pharmacological properties. For instance, compounds containing 2-amino-1,3,4-oxadiazole possess various biological activities such as muscle relaxants $^{(1)}$, anti-mitotics ${ }^{(2)}$, antibacterial and fungicidal ${ }^{(3-5)}$. Also, 1,3,4thiadiazoles exhibit antimicrobial ${ }^{(6)}$ and antitubercular ${ }^{(7-9)}$ activities, while other derivatives act on the CNS as anticonvulsants ${ }^{(10-12)}$ or as antidepressant and anxiolitic $^{(13)}$ agents. Moreover, 1,2,3-triazoles displayed anti-HIV activity ${ }^{(14,15)}$, antimicrobial activity against Gram positive bacteria ${ }^{(16)}$, antiviral $^{(17)}$ and antiproliferative $\mathrm{e}^{(18)}$. The current paper presents an efficient approach for the synthesis of new 1,3,4-oxadiazoles and 1,3,4-thiadiazoles having 1,2,3-triazole nucleus for antimicrobial screening.

\section{Results and Discussion}

\section{Chemistry}

1,2,3-Triazole-4-carbohydrazide (2a-b) were prepared by treatment of 1,2,3triazol-4-ester $(1 \mathrm{a}, \mathrm{b})$ with excess hydrazine hydrate ${ }^{(19)}$. 1-(4-Fluorophenyl)-5methyl-1H-1,2,3-triazole-4-carbohydrazide (2c) was synthesized based on literature procedure ${ }^{(19)}$. Thiosemicarbazides $(3 a-c)$ were prepared in good yields by mixing equimolar amounts of the corresponding hydrazides (2a-c) and phenylisothiocyanate in boiling absolute EtOH for $4 \mathrm{hr}$. 2-(1,2,3-Triazol-4-yl)-

${ }^{\#}$ Corresponding author: E-mail: Bakrfatehy@ yahoo.com 
1,3,4-oxadiazoles (4a-c) were synthesized, instead of product 5, via a desulfurization reaction of thiosemicarbazides (3a-c) with phenacylbromides in ethanol in the presence of anhydrous sodium acetate. Alternatively, 1,3,4oxadiazoles $4 \mathrm{a}-\mathrm{c}$ were prepared by heating $3 \mathrm{a}-\mathrm{c}$ in ethanol in the presence of anhydrous sodium acetate (Scheme 1).

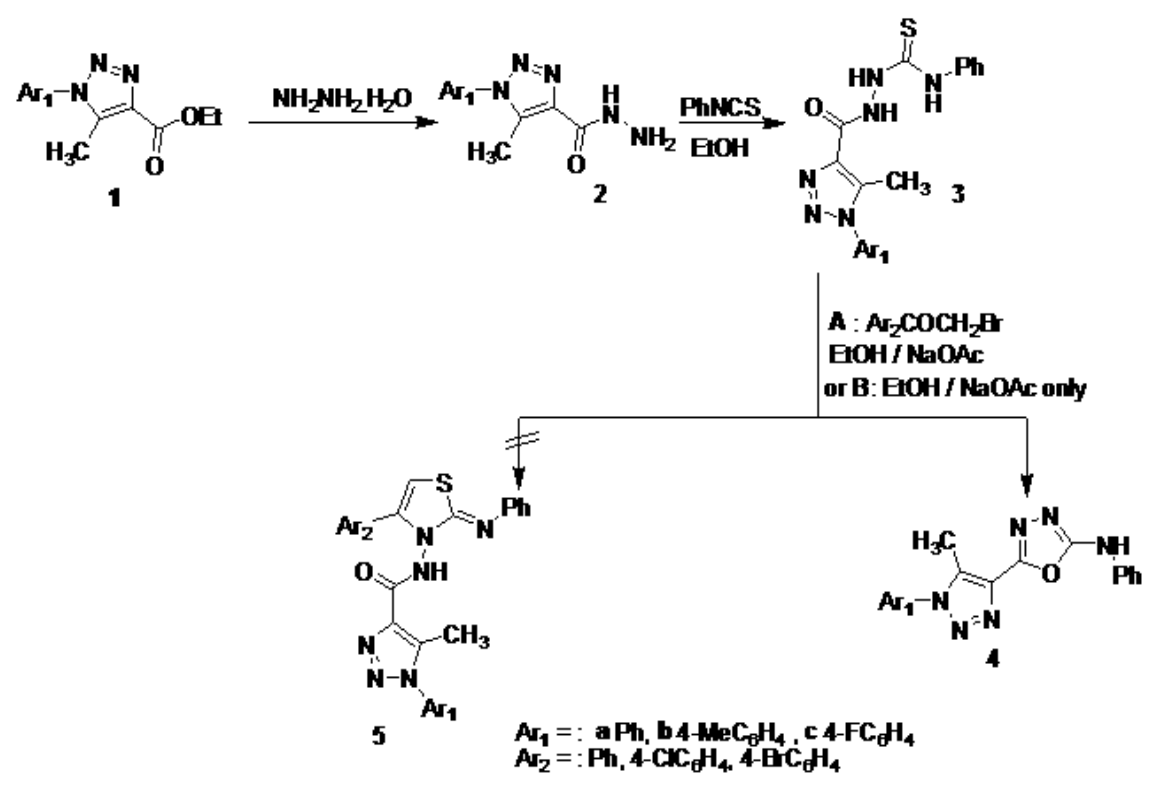

\section{Scheme 1}

${ }^{1} \mathrm{H}$ NMR spectrum of $4 \mathrm{a}-\mathrm{c}$ showed a peak at $10.75 \mathrm{ppm}$ which excluded the formation of 1,3,4-oxadiazol-2-amines not thiazoles $5^{(20)}$. The mass spectrum of $4 \mathrm{c}$ showed the molecular ion peak at $\mathrm{m} / \mathrm{z}$ 336. The structure of $4 \mathrm{~b}$ was confirmed by X-ray crystallography (Fig. 1).

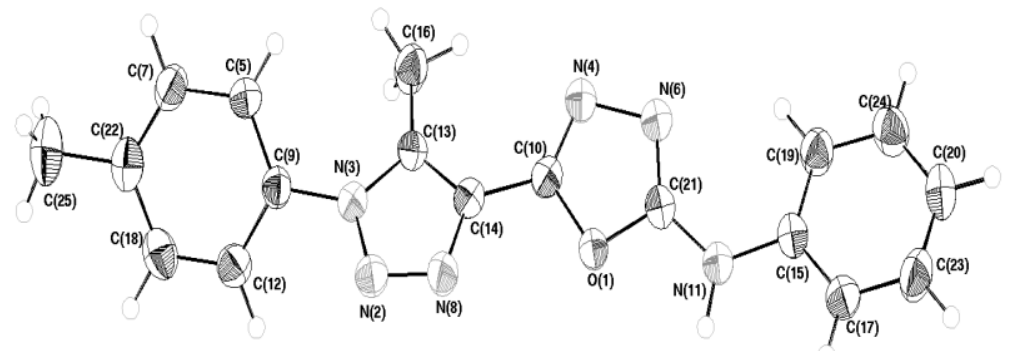

Fig. 1. X-ray structure of compound $4 \mathrm{~b}$.

Egypt. J. Chem. 57, No.4 (2014) 
Treatment of the thiosemicarbazide derivatives 3a-c with cold concentrated sulfuric acid afforded the corresponding 5-(1,2,3-triazol-4-yl)-1,3,4-thiadiazol-2amines (6) (Scheme 2).

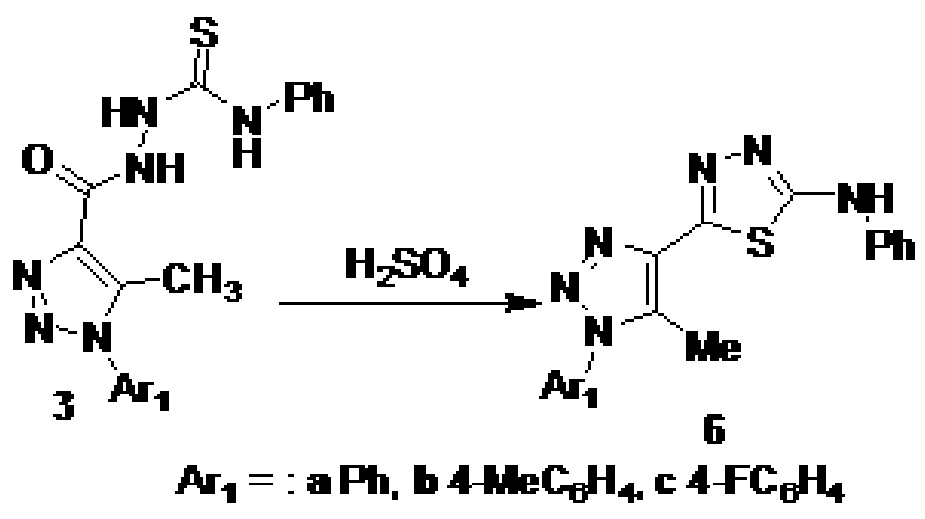

Scheme 2

The ${ }^{1} \mathrm{H}$ NMR spectra of compounds 6a-c indicated the absence of $\mathrm{NH}$ signal as a result of cyclodehydration of 3a-c. The structures of 6a-c were further confirmed by mass spectroscopy. For example, mass spectrum of $6 \mathrm{c}$ showed a molecular ion peak at $\mathrm{m} / \mathrm{z} 352$.

\section{Antimicrobial activity}

The new compounds were screened for their antibacterial activities against three Gram positive bacteria (Staphelococcus aureus ATCC 29213; B. subtilis ATCC6633; and B. megaterium ATCC 9885), three Gram negative bacteria (Klebseilla peneumoniae ATCC13883; Pseudomonas. aeroginosa ATCC27953; and E. coli ATCC 25922) at a concentration $100 \mathrm{~g} / \mathrm{ml}$. Also, the products were tested for their antifungal activities against A. niger and two yeasts (Saccharomyces cervesia and Candida albicans NRRL Y-477). Ciprofloxacin and Ketoconazole were used as standard antibacterial and antifungal reference, respectively. Antimicrobial tests were carried out by disc diffusion method ${ }^{(16)}$. Table 1 summarizes the results of antimicrobial studies, in which compounds 4a-c exhibited a considerably broader antimicrobial activity compared to Ciprofloxacin and Clotrimazole drugs. Products 2c, 3a-c and 6a-c showed good to moderate activity against the tested microorganisms. 
TABLE 1. Antimicrobial activity of products expressed as inhibition diameter zones in millimeters $(\mathrm{mm})$ against the pathological strains based on well diffusion assay.

\begin{tabular}{|c|c|c|c|c|c|c|c|c|c|}
\hline \multirow[b]{2}{*}{$\begin{array}{l}\text { Chem. } \\
\text { Cpds. }\end{array}$} & \multicolumn{3}{|c|}{ Gram positive bacteria } & \multicolumn{3}{|c|}{ Gram negative bacteria } & \multicolumn{2}{|c|}{ Yeast } & \multirow[b]{2}{*}{ 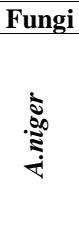 } \\
\hline & 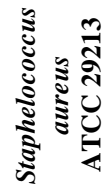 & 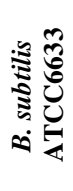 & 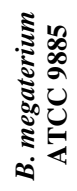 & 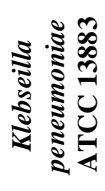 & 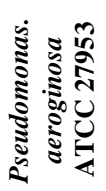 & 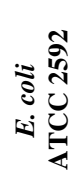 & 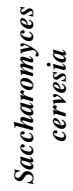 & 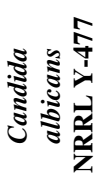 & \\
\hline $2 \mathrm{c}$ & 15 & 18 & 19 & 15 & 15 & N.A. & N.A. & N.A. & 15 \\
\hline $3 a$ & 12 & 16 & 18 & 16 & N.A. & 18 & 17 & 15 & N.A. \\
\hline $3 b$ & 15 & 15 & 16 & 17 & N.A. & 15 & 19 & 18 & 20 \\
\hline $3 \mathrm{c}$ & N.A. & 20 & 22 & 14 & 16 & 16 & 15 & N.A. & N.A. \\
\hline $4 \mathrm{a}$ & 35 & 34 & 32 & 28 & 32 & 28 & 30 & 29 & 28 \\
\hline $4 \mathrm{~b}$ & 35 & N.A. & 12 & 33 & N.A. & 16 & 17 & 30 & 26 \\
\hline $4 \mathrm{c}$ & 36 & 27 & 28 & 38 & 35 & 33 & 34 & 31 & 32 \\
\hline $6 a$ & 15 & 15 & 18 & 17 & 16 & 15 & 18 & 15 & 15 \\
\hline $6 \mathrm{~b}$ & 20 & 15 & 18 & 18 & 15 & 18 & 17 & 15 & 18 \\
\hline $6 \mathrm{c}$ & 20 & 18 & 20 & 18 & 17 & 18 & 20 & 18 & 38 \\
\hline Ciprofloxacin & 20 & 22 & 24 & 28 & 24 & 23 & N.A. & N.A. & N.A. \\
\hline Clotrimazole & N.A. & N.A. & N.A. & N.A. & N.A. & N.A. & 30 & 29 & 30 \\
\hline
\end{tabular}

The experiment was carried out in triplicate and the average zone of inhibition was calculated.

Minimum inhibitory concentration (MIC)

The minimum inhibitory concentration (MIC) of the new products is reported in Table 2. Compounds $2 \mathrm{c}$ and $4 \mathrm{a}$ exhibited the lowest MIC ( $25 \mathrm{~g} / \mathrm{ml})$ against Staphelococcus aureus ATCC 29213. While Compound 4a showed the lowest MIC $(25 \mathrm{~g} / \mathrm{ml})$ against B. subtilis ATCC6633 and Klebseilla peneumoniae ATCC13883, respectively. Compounds $4 \mathrm{c}$ and $6 \mathrm{~b}$ revealed the highest MIC (200 $\mathrm{g} / \mathrm{ml}$ ) against most of the tested bacteria.

TABLE 2. Minimum inhibitory concentration $(\mathrm{mg} / \mathrm{ml})$ against the pathological strains based on two fold serial dilution technique.

\begin{tabular}{|c|c|c|c|c|c|c|c|c|c|}
\hline \multirow[b]{2}{*}{$\begin{array}{l}\text { Chem. } \\
\text { Cpds. }\end{array}$} & \multicolumn{3}{|c|}{ Gram positive bacteria } & \multicolumn{3}{|c|}{ Gram negative bacteria } & \multicolumn{2}{|c|}{ Yeast } & \multirow[b]{2}{*}{ 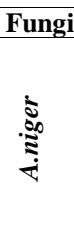 } \\
\hline & 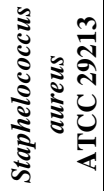 & 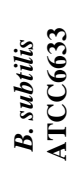 & 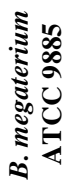 & 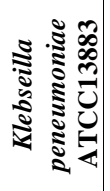 & 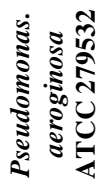 & 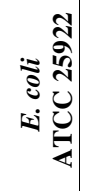 & 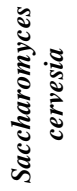 & 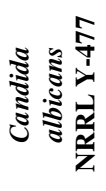 & \\
\hline $2 c$ & 25 & - & - & 50 & - & - & - & 50 & 100 \\
\hline $3 a$ & N.A. & 200 & 100 & N.A. & 200 & 200 & 200 & N.A. & N.A. \\
\hline $3 \mathrm{~b}$ & N.A. & N.A. & 200 & 200 & N.A. & 200 & 200 & N.A. & N.A. \\
\hline $3 \mathrm{c}$ & N.A. & 200 & 100 & N.A. & 200 & 200 & N.A. & N.A. & N.A. \\
\hline $4 a$ & 25 & 25 & 50 & 25 & 32 & 50 & 50 & 50 & 100 \\
\hline $4 \mathrm{~b}$ & N.A. & N.A. & 200 & 200 & 200 & N.A. & 200 & N.A. & N.A. \\
\hline $4 \mathrm{c}$ & 200 & N.A. & 200 & 200 & N.A. & 200 & 200 & N.A. & 200 \\
\hline $6 a$ & N.A. & 200 & 200 & N.A. & N.A. & N.A. & N.A. & N.A. & N.A. \\
\hline $6 \mathrm{~b}$ & 200 & 200 & 200 & 200 & 200 & 200 & 200 & 200 & 25 \\
\hline $6 \mathrm{c}$ & N.A. & N.A. & 200 & 200 & N.A. & N.A. & 200 & 200 & 200 \\
\hline Ciprofloxacin & 25 & 25 & 25 & 25 & 25 & 25 & N.A. & N.A. & N.A. \\
\hline Clotrimazole & N.A. & N.A. & N.A. & N.A. & N.A. & N.A. & 25 & 25 & 25 \\
\hline
\end{tabular}

The experiment was carried out in triplicate and the average zone of inhibition was calculated.

Egypt. J. Chem. 57, No.4 (2014) 


\section{Conclusion}

A facile and efficient approach for the synthesis of 1,2,3-triazol-4-yl-1,3,4oxadiazol-2-amines and 1,2,3-triazol-4-yl-1,3,4-thiadiazoles through direct cyclization reaction of thiosemicarbazides was reported. Compounds $4 \mathrm{a}-\mathrm{c}$ exhibited excellent antimicrobial activity compared to the reference drugs.

\section{Experimental}

\section{Chemistry}

General

All melting points were determined on Electrothermal IA 9000 series digital melting point apparatus. Elemental analytical data were carried out at the Microanalytical Unit, Cairo University, Giza, Egypt. The IR spectra were recorded in potassium bromide disks on a JASCO FT/IR-6100. ${ }^{1} \mathrm{H}-\mathrm{NMR}$ and ${ }^{13} \mathrm{C}$ NMR spectra were run on JOEL-ECA $500 \mathrm{MHz}$ in $\left(\right.$ DMSO- $\left._{6}\right)$. Chemical shifts values $(\delta)$ are given in parts per million (ppm). The mass spectra were performed using mass Varian MAT CH-5 spectrometer at $70 \mathrm{eV}$.

1-(4-Fluorophenyl)-5-methyl-1H-1,2,3-triazole-4-carbohydrazide (2c)

A mixture of 4-acetyl-1,2,3-triazole (1c) $(10 \mathrm{mmol}, 2.5 \mathrm{~g})$ and hydrazine hydrate $(80 \%, 30 \mathrm{mmol}, 1.5 \mathrm{~g})$ in absolute ethanol $(20 \mathrm{ml})$ was heated under reflux for $2 \mathrm{hr}$. The product obtained was collected by filtration, washed with ethanol and dried.

Yield $65 \%$; m.p. $121-123^{\circ} \mathrm{C}$; IR (KBr) max $/ \mathrm{cm}^{-1}, 1670(\mathrm{C}=\mathrm{O})$, 3161-3350 $\left(\mathrm{NH}, \mathrm{NH}_{2}\right) ;{ }^{1} \mathrm{H}$ NMR (DMSO-d $\left.\mathrm{d}_{6}\right) 2.34$ (s, 3H, $\left.\mathrm{CH}_{3}\right), 4.49$ (s, $2 \mathrm{H}, \mathrm{NH}_{2}$ ), 7.31-7.60 (m, 4H, Ar-H), 9.97 (s, $1 \mathrm{H}, \mathrm{NH}, \mathrm{D}_{2} \mathrm{O}$ exchangeable); $\mathrm{MS} \mathrm{m} / \mathrm{z}(\%): 236\left[(\mathrm{M}+1)^{+}\right.$, 18], 235 ( $\left.\mathrm{M}^{+}, 14\right), 91$ (100); Anal. Calcd for $\mathrm{C}_{10} \mathrm{H}_{10} \mathrm{FN}_{5} \mathrm{O}$ (235.22): $\mathrm{C}, 51.06 ; \mathrm{H}$, 4.29; N, 29.77\%. Found: C, 51.13; H, 4.36; N, 29.91\%.

2-(5-Methyl-1-aryl-1H-1,2,3- triazole-4- carbonyl) - N-phenylhydrazinecarbothioamides (3a-c)

A mixture of $2 \mathrm{a}-\mathrm{c}(2 \mathrm{mmol})$ and phenylisothiocyante $(2 \mathrm{mmol}, 0.27 \mathrm{~g})$ in absolute ethanol $(20 \mathrm{ml})$ was heated under reflux for $3 \mathrm{hr}$. The solid obtained was filtered and recrystallized from a mixture of EtOH - DMF (5:1 by volume) to give $3 \mathrm{a}-\mathrm{c}$.

2-(5-Methyl-1-phenyl-1H-1,2,3- triazole-4-carbonyl)- $N$-phenylhydrazinecarbothioamide (3a) : Yield $72 \%$; m.p. $165-166^{\circ} \mathrm{C}$; IR (KBr) $\mathrm{max} / \mathrm{cm}^{-1}$, 1258(C=S), $1660(\mathrm{C}=\mathrm{O}), 3159-3310(\mathrm{NH}) ;{ }^{1} \mathrm{H}$ NMR $\left(\mathrm{DMSO}-\mathrm{d}_{6}\right) 2.34(\mathrm{~s}, 3 \mathrm{H}$, $\left.\mathrm{CH}_{3}\right), 7.27-7.62(\mathrm{~m}, 10 \mathrm{H}, \mathrm{Ar}-\mathrm{H}), 9.46,9.97,10.42\left(3 \mathrm{~s}, 3 \mathrm{H}, 3 \mathrm{NH}, \mathrm{D}_{2} \mathrm{O}\right.$ exchangeable); $\mathrm{MS} \mathrm{m} / \mathrm{z}(\%)$ : $352\left(\mathrm{M}^{+}, 16\right), 91$ (100); Anal. Calcd for $\mathrm{C}_{17} \mathrm{H}_{16} \mathrm{~N}_{6} \mathrm{OS}$ (352.41): C, 57.94; H, 4.58; N, 23.85\%. Found: C, 58.01; H, 4.66; $\mathrm{N}, 23.92 \%$. 
2-(5-Methyl-1-p-tolyl-1H-1,2,3-triazole-4- carbonyl) -N-phenylhydrazinecarbothioamide (3b) : Yield $78 \%$; m.p. $172-174^{\circ} \mathrm{C}$; IR $(\mathrm{KBr}) \mathrm{max} / \mathrm{cm}^{-1}$, 1259(C=S), $1658(\mathrm{C}=\mathrm{O}), 3160-3310(\mathrm{NH}) ;{ }^{1} \mathrm{H}$ NMR $\left(\mathrm{DMSO}^{-\mathrm{d}_{6}}\right)$ 2.34, $2.40(2 \mathrm{~s}$, 6H, $2 \mathrm{CH}_{3}$ ), 7.28-7.63 (m, $\left.9 \mathrm{H}, \mathrm{Ar}-\mathrm{H}\right), 9.45,9.97,10.43$ (3s, 3H, $3 \mathrm{NH}, \mathrm{D}_{2} \mathrm{O}$ exchangeable); MS m/z (\%): $366\left(\mathrm{M}^{+}, 16\right), 91$ (100); Anal. Calcd for $\mathrm{C}_{18} \mathrm{H}_{18} \mathrm{~N}_{6} \mathrm{OS}$ (366.44): C, 59.00; H, 4.95; N, 22.93\%. Found: C, 59.11; H, 4.99; $\mathrm{N}, 23.06 \%$.

2-(1-(4-Fluorophenyl)-5- methyl-1H-1,2,3-triazole-4-carbonyl)-N-phenylhydrazinecarbothioamide $(3 c)$ : Yield $66 \%$; m.p. $205-206^{\circ} \mathrm{C}$; IR $(\mathrm{KBr}) \mathrm{max} / \mathrm{cm}^{-1}$, 1260(C=S), $1659(\mathrm{C}=\mathrm{O}), 3160-3315(\mathrm{NH}) ;{ }^{1} \mathrm{H}$ NMR $\left(\mathrm{DMSO}^{-} \mathrm{d}_{6}\right) 2.35$ (s, 3H, $\left.\mathrm{CH}_{3}\right)$, 7.30-7.67 (m, $\left.9 \mathrm{H}, \mathrm{Ar}-\mathrm{H}\right), 9.44,9.96,10.44\left(3 \mathrm{~s}, 3 \mathrm{H}, 3 \mathrm{NH}, \mathrm{D}_{2} \mathrm{O}\right.$ exchangeable); $\mathrm{MS} \mathrm{m} / \mathrm{z}(\%)$ : $370\left(\mathrm{M}^{+}, 21\right), 91$ (100); Anal. Calcd for $\mathrm{C}_{17} \mathrm{H}_{15} \mathrm{FN}_{6} \mathrm{OS}$ (370.40): C, 55.12; H, 4.08; N, $22.69 \%$. Found: C, 55.30; H, 4.17; $\mathrm{N}, 22.79 \%$.

5-(5-Methyl-1-aryl-1H-1,2,3-triazol-4-yl)-N-phenyl-1,3,4-oxadiazol-2-amines $(4 a-c)$

Method A: A mixture of appropriate 3a-c $(1 \mathrm{mmol})$ and phenacylbromide (1 mmol, $0.2 \mathrm{~g})$ in absolute ethanol $(20 \mathrm{ml})$ containing anhydrous sodium acetate $(2$ mmol, $0.16 \mathrm{~g}$ ) was heated under reflux for $6 \mathrm{hr}$. The reaction mixture was cooled and the formed solid was filtered, washed with water, and recrystallized from a mixture of EtOH - DMF (4:1 by volume).

Method B: A solution of appropriate 3a-c (1 mmol) in absolute ethanol $(20 \mathrm{ml})$ containing anhydrous sodium acetate $(2 \mathrm{mmol}, 0.16 \mathrm{~g})$ was heated under reflux for $6 \mathrm{hr}$. The formed solid was filtered, washed with water, and recrystallized from a mixture of EtOH - DMF (4:1 by volume).

5-(5-Methyl-1-phenyl-1H-1,2,3- triazol-4-yl)- $N$ - phenyl-1,3,4- oxadiazol-2-amine (4a) : Yield $52 \%$; m.p. $215-216^{\circ} \mathrm{C}$; IR (KBr) max $/ \mathrm{cm}^{-1}, 3175(\mathrm{NH}) ;{ }^{1} \mathrm{H}$ NMR (DMSO-d $\left.\mathrm{d}_{6}\right) 2.39\left(\mathrm{~s}, 3 \mathrm{H}, \mathrm{CH}_{3}\right), 6.84-7.51(\mathrm{~m}, 10 \mathrm{H}, \mathrm{Ar}-\mathrm{H}), 10.62(\mathrm{~s}, 1 \mathrm{H}, 1 \mathrm{NH}$, $\mathrm{D}_{2} \mathrm{O}$ exchangeable); $\mathrm{MS} \mathrm{m} / \mathrm{z}(\%): 318\left(\mathrm{M}^{+}, 28\right), 91$ (100); Anal. Calcd for $\mathrm{C}_{17} \mathrm{H}_{14} \mathrm{~N}_{6} \mathrm{O}$ (318.33): C, 64.14; H, 4.43; N, $26.40 \%$. Found: C, 64.28; H, 4.57; N, $26.78 \%$.

5-(5-Methyl-1-p- tolyl-1H-1,2,3-triazol-4-yl)- N-phenyl-1,3,4- oxadiazol-2amine $(4 b)$ : Yield $58 \%$; m.p. $230-231^{\circ} \mathrm{C}$; IR $(\mathrm{KBr}) \mathrm{max} / \mathrm{cm}^{-1}, 3180(\mathrm{NH}) ;{ }^{1} \mathrm{H}$ NMR (DMSO-d $\mathrm{d}_{6}$ ) 2.39, $2.46\left(2 \mathrm{~s}, 6 \mathrm{H}, 2 \mathrm{CH}_{3}\right), 6.82-7.50$ (m, $\left.9 \mathrm{H}, \mathrm{Ar}-\mathrm{H}\right), 10.79$ (s, $1 \mathrm{H}, 1 \mathrm{NH}, \mathrm{D}_{2} \mathrm{O}$ exchangeable); $\mathrm{MS} \mathrm{m} / \mathrm{z}(\%): 332\left(\mathrm{M}^{+}, 32\right), 91$ (100); Anal. Calcd for $\mathrm{C}_{18} \mathrm{H}_{16} \mathrm{~N}_{6} \mathrm{O}$ (332.36): C, 65.05; H, 4.85; N, 25.29\%. Found: C, 65.20; $\mathrm{H}, 4.98 ; \mathrm{N}, 25.63 \%$.

5-(1-(4-Fluorophenyl)-5-methyl-1H-1,2,3-triazol-4-yl)-N-phenyl-1,3,4-oxadiazol2-amine (4c) : Yield $56 \%$; m.p. $258-259^{\circ} \mathrm{C}$; IR (KBr) max $/ \mathrm{cm}^{-1} 3183(\mathrm{NH}) ;{ }^{1} \mathrm{H}$ NMR (DMSO-d $\left.\mathrm{d}_{6}\right) 2.36\left(\mathrm{~s}, 3 \mathrm{H}, \mathrm{CH}_{3}\right), 6.81-7.54(\mathrm{~m}, 9 \mathrm{H}, \mathrm{Ar}-\mathrm{H}), 10.67(\mathrm{~s}, 1 \mathrm{H}, 1$

Egypt. J. Chem. 57, No.4 (2014) 
$\mathrm{NH}, \mathrm{D}_{2} \mathrm{O}$ exchangeable); ${ }^{13} \mathrm{C}$ NMR (DMSO-d $\mathrm{d}_{6}$ ) 10.9, 111, 112, 116.3, 118, 122, $125,128,129.8,136.9,139,148,154,162 ; \mathrm{MS} \mathrm{m} / \mathrm{z}(\%): 336\left(\mathrm{M}^{+}, 28\right), 91$ (100); Anal. Calcd for $\mathrm{C}_{17} \mathrm{H}_{13} \mathrm{FN}_{6} \mathrm{O}$ (336.32): C, 60.71; H, 3.90; N, $24.99 \%$. Found: C, 60.82; H, 3.99; N, 25.11\%.

5-(5-Methyl-1-aryl-1H-1,2,3-triazol-4-yl)- N-phenyl-1,3,4-thiadiazol-2-amine
(6a-c)

A suspension of appropriate $3 \mathrm{a}-\mathrm{c}(1 \mathrm{mmol})$ in concentrated sulfuric acid $(5 \mathrm{ml})$ was stirred in an ice bath for $1 \mathrm{hr}$. The reaction mixture was left to stand overnight, then poured into ice-water and neutralized with $2 \mathrm{~N} \mathrm{NaOH}$. The formed solid was filtered, dried, and crystallized from ethanol.

5-(5-Methyl-1- phenyl-1H-1,2,3-triazol-4-yl)- $N$-phenyl-1,3,4-thiadiazol-2-amine (6a) : Yield $68 \%$; m.p. $207-209^{\circ} \mathrm{C}$; IR (KBr) max $/ \mathrm{cm}^{-1} 3220(\mathrm{NH}) ;{ }^{1} \mathrm{H}$ NMR (DMSO-d $\left.\mathrm{d}_{6}\right) \quad 2.40\left(\mathrm{~s}, 3 \mathrm{H}, \mathrm{CH}_{3}\right), 7.34-7.65(\mathrm{~m}, 10 \mathrm{H}, \mathrm{Ar}-\mathrm{H}), 10.62(\mathrm{~s}, 1 \mathrm{H}, 1 \mathrm{NH}$, $\mathrm{D}_{2} \mathrm{O}$ exchangeable); $\left.\mathrm{MS} \mathrm{m} / \mathrm{z}(\%): 335\left[(\mathrm{M}+1)^{+}, 34\right)\right], 334\left(\mathrm{M}^{+}, 22\right), 91(100)$; Anal. Calcd for $\mathrm{C}_{17} \mathrm{H}_{14} \mathrm{~N}_{6} \mathrm{~S}$ (334.40): C, 61.06; H, 4.22; N, $25.13 \%$. Found: $\mathrm{C}$, 61.32; H, 4.66; N, 25.33\%.

5-(5-Methyl-1-p- tolyl-1H-1,2,3-triazol-4-yl)- $N$ - phenyl-1,3,4- thiadiazol-2amine (6b) : Yield $64 \%$; m.p. $268-269^{\circ} \mathrm{C}$; IR (KBr) max $/ \mathrm{cm}^{-1} 3220(\mathrm{NH}) ;{ }^{1} \mathrm{H}$ NMR (DMSO-d $\mathrm{d}_{6}$ ) 2.40, $2.47\left(2 \mathrm{~s}, 6 \mathrm{H}, 2 \mathrm{CH}_{3}\right), 7.30-7.64(\mathrm{~m}, 9 \mathrm{H}, \mathrm{Ar}-\mathrm{H}), 10.63$ (s, $1 \mathrm{H}, 1 \mathrm{NH}, \mathrm{D}_{2} \mathrm{O}$ exchangeable); $\left.\mathrm{MS} \mathrm{m} / \mathrm{z}(\%): 349\left[(\mathrm{M}+1)^{+}, 32\right)\right], 348\left(\mathrm{M}^{+}, 22\right)$, 91 (100); Anal. Calcd for $\mathrm{C}_{18} \mathrm{H}_{16} \mathrm{~N}_{6} \mathrm{~S}$ (348.42): C, 62.05; H, 4.63; N, 24.12\%. Found: C, 62.61; H, 4.73; N, 24.32\%.

5-(1-(4-Fluorophenyl)-5-methyl-1H-1,2,3-triazol-4-yl)-N-phenyl-1,3,4-thiadiazol2-amine (6c) : Yield $58 \%$; m.p. $315-317^{\circ} \mathrm{C}$; IR $(\mathrm{KBr}) \mathrm{max} / \mathrm{cm}^{-1} 3220(\mathrm{NH}) ;{ }^{1} \mathrm{H}$ NMR (DMSO-d $\left.{ }_{6}\right) 2.40\left(\mathrm{~s}, 3 \mathrm{H}, \mathrm{CH}_{3}\right), 7.32-7.65(\mathrm{~m}, 9 \mathrm{H}, \mathrm{Ar}-\mathrm{H}), 10.67(\mathrm{~s}, 1 \mathrm{H}, 1$ $\mathrm{NH}, \mathrm{D}_{2} \mathrm{O}$ exchangeable); ${ }^{13} \mathrm{C}$ NMR (DMSO-d $\mathrm{d}_{6}$ ) $10.9,111,115,117,122,123$, 125, 128.6, 131, 133, 136.7, 144, 153.2, 162; MS m/z (\%): 353 [(M+1) $\left.\left.{ }^{+}, 38\right)\right]$, $352\left(\mathrm{M}^{+}, 24\right), 91$ (100); Anal. Calcd for $\mathrm{C}_{17} \mathrm{H}_{13} \mathrm{FN}_{6} \mathrm{~S}$ (352.39): C, 57.94; H, 3.72; N, 23.85\%. Found: C, 58.11; H, 3.84; N, $25.08 \%$.

Antimicrobial activity

Antimicrobial tests were carried out by the agar well diffusion method ${ }^{(16)}$.

Minimal inhibitory concentration (MIC) measurement

The bacteriostatic activity of the active compounds was evaluated using the two fold serial dilution technique ${ }^{(16)}$.

$X$-ray crystallography

A single crystal of compound $4 \mathrm{~b}$ was obtained by slow evaporation at room temperature, from dimethylformamide (DMF). The crystal structure was solved and refined using MaXus (Bruker Nonius, Deflt and MacScience, Japan) ${ }^{(21)}$. Mo $\mathrm{K}_{\alpha}$ radiation $(\lambda=0.71073 \AA \hat{)})$ and a graphite monochromator were used for data 
collection. The chemical formula and ring labeling system is shown in Fig. 1. Crystal data for compound $4 \mathrm{~b}: \mathrm{C}_{18} \mathrm{H}_{16} \mathrm{~N}_{6} \mathrm{O}, \mathrm{Mr}, 332.367$; system, orthorhombic; Space group, $\mathrm{P} 2{ }_{1} 2_{1} 2_{1}$; unit cell dimensions, a, 6.4629 (2) $\AA$; b, 10.7257 (4) $\AA$; c, 12.5359 (4) $\AA ; \alpha, 108.565(2)^{\circ} ; \beta, 99.650(2)^{\circ} ; \gamma, 95.2020(13)^{\circ} ; \mathrm{V}, 802.40(5) \AA^{3}$; $\mathrm{Z}, 2 ; \mathrm{D}_{\mathrm{x}}, 1.376 \mathrm{Mg} \mathrm{m}^{-3} ; \theta_{\max }=30.07^{\circ} ; \mu\left(\right.$ Mo- $\left.K_{\alpha}\right), 0.09 \mathrm{~mm}^{-1} ; \mathrm{T}=298 \mathrm{~K}$; independent reflections, 5257; measured reflections, 11255; observed reflections, 2389; $\mathrm{R}_{\text {int }}, 0.050$; $\mathrm{R}(\mathrm{all}), 0.124$; $\mathrm{R}(\mathrm{gt}), 0.050$; $\mathrm{wR}(\mathrm{ref}), 0.095$; wR(all), 0.109; wR(gt), 0.095; S(ref), 0.719; S(all), 0.683; S(gt), 0.721; $\Delta / \sigma_{\max }, 0.013, \Delta \rho_{\max }$, $0.39 \mathrm{e}^{3} ; \Delta \rho_{\min }-0.36 \mathrm{e}^{3}$.

Crystallographic data for the structures $4 \mathrm{~b}$ have been deposited at the Cambridge Crystallographic Data Center (CCDC) under the number 993032. Copies of the data can be obtained, free of charge, on application to CCDC 12 Union Road, Cambridge CB2 1EZ, UK [Fax: +44-1223-336033; e-mail: deposit@ ccdc.cam.ac.uk or at www.ccdc.cam.ac.uk].

\section{References}

1. Yale, H.L. and Losee, K., 2-Amino-5-substituted 1,3,4-oxadiazoles and 5-imino-2substituted $82-1,3,4-$ oxadiazolines. A group of novel muscle relaxants. J. Med. Chem. 9, 478 (1966).

2. Kiselyov, A.S., Semenova, M.N., Chernyshova, N.B., Leitao, A., Samet, A.V., Kislyi, K.A., Raihstat, M.M., Oprea, T., Lemcke, H. and Lantow, M., Novel derivatives of 1,3,4-oxadiazoles are potent mitostatic agents featuring strong microtubule depolymerizing activity in the sea urchin embryo and cell culture assays. Eur. J. Med. Chem. 45, 1683 (2010).

3. Khan, M.T.H., Choudhary, M.I., Khan, K.M. and Rani, M., Structure-activity relationships of tyrosinase inhibitory combinatorial library of 2,5-disubstituted-1,3,4oxadiazole analogues. Bioorg. Med. Chem. 13, 3385 (2005).

4. Ramazani, A. and Rezaei, A., Novel one-pot, four-component condensation reaction: an efficient approach for the synthesis of 2,5-disubstituted 1,3,4-oxadiazole derivatives by a Ugi-4CR/aza-Wittig sequence. Org. Lett. 12, 2852 (2010).

5. Brown, P., Best, D.J., Broom, N.J.P., Cassels, R., O'Hanlon, P.J., Mitchell, T.J., Osborne, N.F. and Wilson, J.M., The chemistry of pseudomonic acid. 18. heterocyclic replacement of the $\alpha, \beta$-unsaturated ester: Synthesis, molecular modeling, and antibacterial activity. J. Med. Chem. 40, 2563 (1997).

6. Dogan, H.N., Duran, A., Rollas, S., Sener, G., Uysal, M.K. and Gulen, D., Synthesis of new 2,5-disubstituted-1,3,4-thiadi azoles and preliminary evaluation of anticonvulsant and antimicrobial activities. Bioorg. Med. Chem. Lett. 10, 2893 (2002).

7. Mamolo, M.G., Falagiani, V., Zampieri, D., Vio, L., Banfi, E. and Scialino, G., Synthesis and antimycobacterial activity of (3,4-diaryl-3H-thiazol-2-ylidene)hydrazide derivatives. Farmaco, 58, 631 (2003). 
8. Foroumadi, A., Kiani, Z. and Soltani, F., Antituberculosis agents VIII-Synthesis and in vitro antimycobacterial activity of alkyl $\alpha$-[5-(5-nitro-2-thienyl)-1,3,4thiadiazole-2-ylthio] acetates. Farmaco, 58, 1073 (2003).

9. Oruc, E.E., Rollas, S., Kandemirli, F., Shvets, N. and Dimoglo, A.S., 1,3,4Thiadiazole derivatives. Synthesis, structure elucidation, and structure-antituberculosis activity relationship investigation. J. Med. Chem. 47, 6760 (2004).

10. Stillings, M.R., Welbourn, A.P. and Walter, D.S., Substituted 1,3,4-thiadiazoles with anticonvulsant activity. 2. Aminoalkyl derivatives. J. Med. Chem. 29, 2280 (1986).

11. Chapleo, C.B., Myers, P.L., Smith, A.C., Tulloch, I.F. and Walter, D.S., Substituted 1,3,4-thiadiazole with anticonvulsant activity. J. Med. Chem. 30, 951 (1987).

12. Chimirri, A., Grasso, S., Monforte, A.M. and Zappala, M., Synthesis and anticonvulsant properties of 3-(1,3,4-thiadiazol-2-yl)thiazolidin-4-ones. Farmaco, 46, 935 (1991).

13. Clerici, F., Pocar, D., Maddalena, G., Loche, A., Perlini, V. and Brufani, M., Synthesis of 2-amino-5-sulfanyl-1,3,4-thiadiazole derivatives and evaluation of their antidepressant and anxiolytic activity. J. Med. Chem. 44, 931 (2001).

14.Lazrek, H.B., Taourirte, M., Oulih, T., Barascut, J.L., Imbach, J.L., Pannecouque, C., Witrouw, M. and De Clercq, E., Synthesis and anti-HIV activity of new modified 1,2,3-triazole acyclonucleosides. Nucleosides Nucleotides Nucleic Acids, 20 , 1949 (2001).

15. Velazquez, S., Alvarez, R., Perez, C., Gago, F., De, C., Balzarini, J. and Camarasa, M.J., Regiospecific synthesis and anti-human immunodeficiency virus activity of novel 5-substituted N-alkylcarbamoyl and N,N-dialkylcarbamoyl 1,2,3triazole-TSAO analogues. Antivir. Chem. Chemother. 9, 481 (1998).

16. Abdel-Wahab, B.F., Abdel-Latif, E., Mohamed, H.A. and Awad,G.E.A., Design and synthesis of new 4-pyrazolin-3-yl-1,2,3-triazoles and 1,2,3-triazol-4-yl-pyrazolin1 -ylthiazoles as potential antimicrobial agents. Eur. J. Med. Chem. 52, 263 (2012); Abdel-Wahab, B.F., Sediek, A., Mohamed, H.A. and Awad, G.E.A., Novel 2pyrazolin-1-ylthiazoles as potential antimicrobial agents. Letters in Drug Design and Discovery, 10, 111 (2013).

17. Jordão, A.K., Ferreira, V.F., Souza, T.M., Faria, G.G., Machado, V., Abrantes, J.L., de Souza, M.C. and Cunha, A.C., Synthesis and anti-HSV-1 activity of new 1,2,3-triazole derivatives. Bioorg. Med. Chem. 19, 1860 (2011).

18. Duan, Y.C., Zheng, Y.C., Li, X.C., Wang, M.M., Ye, X.W., Guan, Y.Y., Liu, G.Z., Zheng, J.X. and Liu, H.M., Design, synthesis and antiproliferative activity studies of novel 1,2,3-triazole-dithiocarbamate-urea hybrids. Eur. J. Med. Chem. 64, 99 (2013).

19. Zhang, Y., Qiao, R.Z., Xu, P.F., Zhang, Z.Y., Wang, Q., Mao, L.M. and Yu, K.B., Synthesis and antibacterial activities of 2-(1-aryl-5-methyl-1,2,3-triazol-4-yl)-1,3,4oxadiazole derivatives. J. Chin. Chem. Soc. 49, 369 (2002). 
20. Maghari, S., Ramezanpour, S., Darvish, F., Balalaie, S., Rominger, F. and Bijanzadeh, H.R., A new and efficient synthesis of 1,3,4-oxadiazole derivatives using TBTU. Tetrahedron, 69, 2075 (2013).

21. Mackay, S., Gilmore, C. J., Edwards, C., Stewart, N. and Shankland, K., maXus Computer Program for the Solution and Refinement of Crystal Structures. Bruker Nonius, The Netherlands, MacScience, Japan \& The University of Glasgow (1999).

(Received 11/8/2014; accepted 20/8/2014)

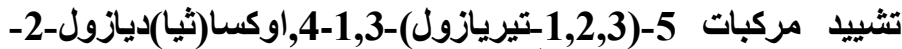

$$
\begin{aligned}
& \text { امين الجديدة كمضادات للفطريات و البكتيريا }
\end{aligned}
$$

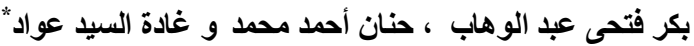

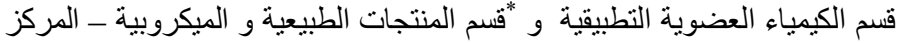

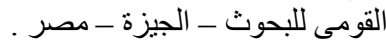

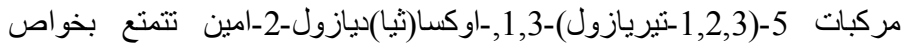

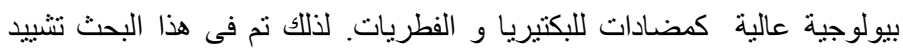

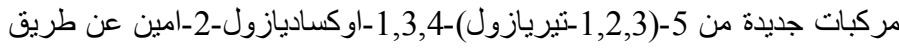

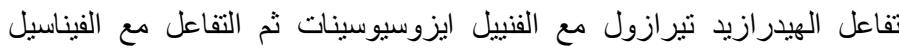

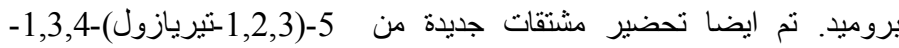

ثياديازول-2-امين عن طريق تفاعل الثيو سيمى كبرزيد مع حمض الكبرتئ منيك

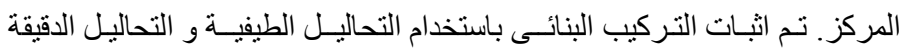

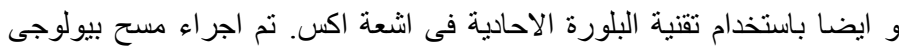

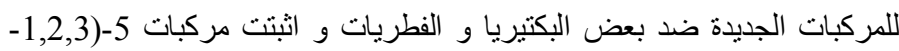

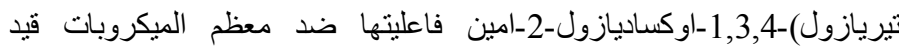

$$
\text { المسح. ايضا نم اجر اء اختبار اقل تركيز لمنع نمو الميكروبات. }
$$

Egypt. J. Chem. 57, No.4 (2014) 